\title{
The Closed-Form Solution of the Reduced Fokker-Planck-Kolmogorov Equation for Nonlinear Systems
}

\author{
Lincong Chen ${ }^{\mathrm{a}}$ Jian-Qiao Sun ${ }^{\mathrm{b}}$ \\ ${ }^{a}$ College of Civil Engineering, Huaqiao University \\ Xiamen, Fujian, 361021, China \\ ${ }^{\mathrm{b}}$ School of Engineering, University of California \\ Merced, CA 95343, USA \\ Corresponding author: J.Q. Sun (jqsun@ucmerced.edu)
}

\begin{abstract}
In this paper, we propose a new method to obtain the closed-form solution of the reduced Fokker-Planck-Kolmogorov equation for single degree of freedom nonlinear systems under external and parametric Gaussian white noise excitations. The assumed stationary probability density function consists of an exponential polynomial with a logarithmic term to account for parametric excitations. The undetermined coefficients in the assumed solution are computed with the help of an iterative method of weighted residue. We have found that the iterative process generates a sequence of solutions that converge to the exact solutions if they exist. Three examples with known exact steady-state probability density functions are used to demonstrate the convergence of the proposed method.
\end{abstract}

Key words: closed-form solution; steady-state probability density function; weighted residue method; iterative technique; random vibrations; nonlinear dynamics.

\section{Introduction}

Dynamic loadings on structures such as those due to winds, sea waves, earthquake, roadway irregularities, rocket are random in nature. These loadings can be strong enough to cause nonlinear responses of the structure. When the excitations are modeled as the Gaussian white noise, the response of the structure forms a Markov process whose response statistics is completely characterized by the probability density function governed by the FokkerPlanck-Kolmogorov (FPK) equation. There had been strong interests in finding solutions 
of FPK equations for nonlinear systems. The major progresses were well documented in the seminal book by Cai and Lin [1]. The book contains a very comprehensive selection of nonlinear stochastic systems for which the exact solutions of the reduced FPK equation had been obtained. There have been much less results of exact solutions reported since then. This paper presents an iterative method that can construct highly accurate solutions of the reduced FPK equations, and converge to the exact solutions of the reduced FPK equation for all the cases reported in [1].

Researchers have long had a strong interest in finding the exact solutions of the FPK equation. The exact solutions of the FPK equation can be found for a very few one dimensional cases $[2,3]$. For the two- and higher-dimensional systems, the exact solutions of the reduced FPK equation have obtained under strict conditions by Caughey and Fai [4], Dimentberg [5],Yong and Lin [6], Cai and Lin [1], and Zhu and his coworkers [7,8]. The concept of detailed balance is a popular approach used to construct the exact solution of the reduced FPK equation for nonlinear systems under both additive and multiplicative white noise excitations. Indeed, many examples of the exact solutions can be found in the framework of the detailed balance. In 1990s, Lin and Cai [9] developed a systematic procedure using the probability potential and detailed balance of the generalized probability potential to obtain exact solutions of the reduced FPK equation for single- and multi-degree-of-freedom nonlinear oscillatory systems. There has been no new progress on the exact solution of the reduced FPK equation since then.

Since there is a limited number of nonlinear stochastic systems for which we can obtain the exact solution of the FPK equation, we must develop procedures to compute the solution numerically or analytically.

In the literature, various approximate methods have been developed to solve the FPK equation, including the finite element method [10-15] , finite difference method [16, 17], path integral [18-20], iterative scheme [21,22], the principle of maximum entropy [23-25], eigenfunction-expansion [26-28], variational method [29] and method of weighted residue [30-36]. These methods have limitations. For example, the finite element and finite difference are computationally intensive. The path integration is feasible for the low dimensional systems. The solution obtained by the method of weighted residue is influenced by the weighting functions. More comments on these numerical methods were given in the review article [37] and a monograph [38].

In the last decade, Er proposed the method of exponential polynomial closure (EPC) to solve the reduced FPK equation [33-35]. In this method, the steady-state PDF is approximated as an exponential function of a polynomial in state variables with undetermined coefficients. The assumed solution satisfies the reduced FPK equation in the weak sense. The undetermined coefficients of the polynomial are determined by solving simultaneous quadratic algebraic equations derived from the method of weighted residue. Recently, the EPC method has been extended to the nonlinear systems excited by a Poisson white noise [39] or by a combined Gaussian and Poisson white noises [40]. They have studied the system with multiple peaks in the PDF [41], and a nonlinear impact oscillator $[42,43]$. In conjunction with the 
method of sub-space-slice, the EPC method has been used to predict the steady-state PDF of multi-degree-of-freedom nonlinear systems [44]. De Paola and Sofi [45] generalized Er's EPC method with the help of a proper choice of the weighting functions, and suggested a simple and effective iterative procedure to improve the accuracy of the approximate solution.

In the current paper, an iterative method for the closed-form solution of the steady-state PDF of single degree of freedom nonlinear systems under external and parametric Gaussian white noise excitations is developed. The proposed method can obtain the exact steady-state PDF of the system, if it exists, starting from a weighting function in the form of Gaussian PDF or an approximate PDF obtained with the method of stochastic averaging, for example.

The rest of the paper is outlined as follows. Section 2 outlines the steps of the proposed methods. It starts with the reduced FPK equation, then applying the method of weighted residue to determine the undetermined coefficients of the assumed solution in terms of the exponential function of polynomials in the state variables plus a logarithmic term in Section 2.1. Section 2.3 introduces an iterative procedure to improve the accuracy of the solutions obtained with the method of weighted residue. Section 2.4 presents a progressively iterative procedure for strongly nonlinear systems. In Section 3, three examples with known exact solutions of the reduced FPK equation are studied to demonstrate the convergence of the iterative procedure. The accuracy of the approximate PDF is measured against the exact solution or the Monte Carlo simulations. Section 4 concludes the paper.

\section{The Proposed Method}

The proposed method to construct the analytical solution of the reduced FPK equation consists of three steps. We apply the method of weighted residue for determining the unknown parameters in the assumed solution, and propose an iterative procedure to improve the accuracy of the solution obtained with the method of weighted residue. As an extension of the method, we further propose a progressively iterative procedure in the nonlinear parameter space to obtain the PDFs for highly nonlinear stochastic systems.

\subsection{The Reduced FPK Equation}

Consider a Stratonovich stochastic differentiation equation for a single degree of freedom nonlinear oscillator under external and parametric Gaussian white noise excitations in the state space form as

$$
\begin{aligned}
& \frac{d X}{d t}=Y, \\
& \frac{d Y}{d t}=-g(X, Y)+\sum_{i=1}^{l}\left[h_{i}(X, Y)\right] W_{i}(t),
\end{aligned}
$$


where $g(X, Y)$ includes the damping and restoring forces, $h_{i}(X, Y)$ are linear or nonlinear functions, $W_{i}(t)$ denote independent Gaussian white noises with intensities $2 D_{i j}(i, j=$ $1,2, \ldots, l)$.

The reduced FPK equation of the system reads,

$$
\mathcal{L}[p] \equiv-\frac{\partial}{\partial x}\left[p m_{1}\right]+\frac{\partial}{\partial y}\left[p m_{2}\right]+\frac{\partial^{2}}{\partial y^{2}}\left[p b_{22}\right]=0
$$

where $\mathcal{L}[p]$ denotes the differential operator of the FPK equation, $p=p(x, y)$, the drift and diffusion terms $m_{1}, m_{2}$ and $b_{22}$ are given by

$$
\begin{aligned}
& m_{1}=y, \\
& m_{2}=g(x, y)-\sum_{i=1}^{l} \sum_{j=1}^{l}\left[D_{i j} \frac{\partial h_{i}(x, y)}{\partial y} h_{j}(x, y)\right], \\
& b_{22}=\sum_{i=1}^{l} \sum_{j=1}^{l}\left[D_{i j} h_{i}(x, y) h_{j}(x, y)\right] .
\end{aligned}
$$

Based on the observations of the existing closed-form solutions of the reduced FPK equation for the systems under external and parametric excitations, we propose the solution of Equation (2) to be in the following form,

$$
\bar{p}(x, y)=C_{0} \exp \left[\varphi\left(x, y, c_{i j}\right)+k_{0} \ln \left(b_{22}\right)\right]
$$

where $C_{0}$ is a normalization constant, $c_{i j}$ and $k_{0}$ are the undetermined parameters, the logarithmic term $\ln \left(b_{22}\right)$ accounts for the parametric excitations, and $\varphi\left(x, y, c_{i j}\right)$ represents an $n^{\text {th }}$ order polynomial given by

$$
\varphi\left(x, y, c_{i j}\right)=\sum_{i, j \geq 0} \sum_{0<i+j \leq n} c_{i j} x^{i} y^{j}
$$

Where there is no parametric excitation, $b_{22}$ is a constant. In this case, the whole logarithmic term $k_{0} \ln \left(b_{22}\right)$ is absorbed into the normalization constant $C_{0}$.

The conditions for the existence of $\bar{p}(x, y)$ are stated as

$$
\begin{gathered}
\varphi\left(x, y, c_{i j}\right)+k_{0} \ln \left(b_{22}\right) \rightarrow-\infty \text { as } A \rightarrow \infty, \forall \theta \in[0,2 \pi], \\
\alpha>-1 \text { if }\left|b_{22}^{k_{0}}\right| \rightarrow A^{\alpha} \text { as } A \rightarrow 0,
\end{gathered}
$$

where $x=A \cos \theta$ and $y=A \sin \theta$. The second condition is for the integrability of $\bar{p}(x, y)$ at the origin. 


\subsection{Method of Weighted Residue}

Inserting Equation (4) into Equation (2) yields a residual error $\mathcal{L}[\bar{p}]=r\left(x, y, c_{i j}, k_{0}\right) \bar{p}(x, y)$ where

$$
\begin{aligned}
r\left(x, y, c_{i j}, k_{0}\right) & =m_{1}\left[\frac{\partial \varphi}{\partial x}+\frac{k_{0}}{b_{22}} \frac{\partial b_{22}}{\partial x}\right]+\frac{\partial m_{2}}{\partial y}- \\
& +m_{2}\left[\frac{\partial \varphi}{\partial y}+\frac{k_{0}}{b_{22}} \frac{\partial b_{22}}{\partial y}\right]+\frac{\partial^{2} \varphi}{\partial y^{2}}+\frac{k_{0}}{b_{22}} \frac{\partial^{2} b_{22}}{\partial y^{2}} \\
& -\frac{k_{0}}{b_{22}^{2}}\left(\frac{\partial b_{22}}{\partial y}\right)^{2}+\left[\frac{\partial \varphi}{\partial y}+\frac{k_{0}}{b_{22}} \frac{\partial b_{22}}{\partial y}\right]^{2} \\
& +2\left[\frac{\partial \varphi}{\partial y}+\frac{k_{0}}{b_{22}} \frac{\partial b_{22}}{\partial y}\right] \frac{\partial b_{22}}{\partial y}+\frac{\partial^{2} b_{22}}{\partial y^{2}}
\end{aligned}
$$

We apply the method of weighted residue, a global minimization scheme, to find the undetermined coefficients. Let $M_{i_{1} j_{1}}(x, y)$ be a set of weighting functions. We impose the following conditions,

$$
\int_{-\infty}^{+\infty} \int_{-\infty}^{+\infty} M_{i_{1} j_{1}}(x, y) r\left(x, y, c_{i j}, k_{0}\right) d x d y=0
$$

where the indices $i_{1}$ and $j_{1}$ of $M_{i_{1} j_{1}}(x, y)$ are in the range of $0 \leq i_{1}+j_{1} \leq n$. Recall that for $c_{i j}, 0<i+j \leq n$. Hence, the number of weighting functions is one more than the number of the coefficients $c_{i j}$. The weighting function with $i_{1}=j_{1}=0$ provides an equation for $k_{0}$. We note that since $\bar{p}(x, y)>0$ for all $x$ and $y$, we omit it from the weighted residue evaluation in Equation (8). This substantially simplifies the computations for the solutions of $c_{i j}$ and $k_{0}$. The reduced FPK equation (2) is satisfied with $\bar{p}(x, y)$ in the weak sense if the integration in Equation (8) can be solved numerically.

A key step of the method of weighted residue is to select a set of weighting functions $M_{i_{1} j_{1}}(x, y)$ that lead to a set of nonlinear algebraic equations to determine the parameters $c_{i j}$ and $k_{0}$.

Consider a set of weighting functions in the following form,

$$
M_{i_{1} j_{1}}(x, y)=p_{m}(x, y) x^{i_{1}} y^{j_{1}}
$$

where $p_{m}(x, y)$ can be a Gaussian PDF or an approximate PDF obtained with the method of stochastic averaging, for example. The choice of the weighting function in Equation (9) implies that we treat $x^{i_{1}} y^{j_{1}}$ as the base of the polynomials in the solution (4), and require the zero residual error projected on to this base.

The integration domain in Equation (8) must be finite in numerical studies. We have used rough Monte Carlo simulation results to estimate a finite integration domain $\Omega_{s}$ such that 
at least $95 \%$ of the probability of the system is contained in it. Equation (8) is then approximated by

$$
\iint_{\Omega_{s}} M_{i_{1} j_{1}}(x, y) r\left(x, y, c_{i j}, k_{0}\right) d x d y=0 .
$$

On the other hand, if $p_{m}(x, y)$ is Gaussian, the integration domain can be taken as $\left[-4 \sigma_{x}, 4 \sigma_{x}\right] \times$ $\left[-4 \sigma_{y}, 4 \sigma_{y}\right]$ where $\left(\sigma_{x}, \sigma_{y}\right)$ are the standard deviations of $x$ and $y$.

Equation (10) leads to a set of nonlinear algebraic equations which can be solved numerically. The solutions of $c_{i j}$ and $k_{0}$ will be accepted if the existence condition (6) is satisfied. Otherwise, the solution will be discarded.

\subsection{Iterative Procedure}

The application of the method of weighted residue just once may not obtain sufficiently accurate results, particularly for poorly selected weighting functions. Here, we propose an iterative procedure similar to the method proposed in [45] to improve the accuracy of the solutions.

Let $\bar{p}^{(k)}$ be the approximation of the steady-state PDF after applying the method of weighted residue $k$ times. We use $\bar{p}^{(k)}$ in place of $p_{m}(x, y)$ in Equation (9) for all $k>0$ to compute the next approximation of the PDF denoted as $\bar{p}^{(k+1)}$ with the method of weighted residue. We repeat these steps until the following convergence criterion is satisfied

$$
\varepsilon=\sqrt{\int_{-\infty}^{+\infty} \int_{-\infty}^{+\infty}\left(\bar{p}^{(k)}-\bar{p}^{(k+1)}\right)^{2} d x d y} \approx \sqrt{\iint_{\Omega_{s}}\left(\bar{p}^{(k)}-\bar{p}^{(k+1)}\right)^{2} d x d y} \leqslant \varepsilon_{0}
$$

where $\varepsilon_{0}$ is a preset tolerance. We check in every iteration if the existence condition (6) is satisfied. Numerical computations indicate that the convergence of the iterative procedure is quite fast. Other convergence criterions can be defined. For example, a residual error based criterion can be defined as,

$$
\varepsilon=\sqrt{\int_{-\infty}^{+\infty} \int_{-\infty}^{+\infty}\left(r \bar{p}^{(k)}\right)^{2} d x d y} \approx \sqrt{\iint_{\Omega_{s}}\left(r \bar{p}^{(k)}\right)^{2} d x d y} \leqslant \varepsilon_{0}
$$

In order to evaluate the accuracy of the converged solution, we define the error of the 
computed PDF with respect to a reference solution denoted as $p_{R}(x, y)$,

$$
\begin{aligned}
\Delta p_{k} & =\sqrt{\iint_{\Omega_{s}}\left(\bar{p}^{(k)}-p_{R}\right)^{2} d x d y} \\
& \approx \sqrt{\sum_{i=0}^{N_{1}} \sum_{j=0}^{N_{2}}\left[\bar{p}^{(k)}\left(x_{i}, y_{j}\right)-p_{R}\left(x_{i}, y_{j}\right)\right]^{2} \Delta x \Delta y}
\end{aligned}
$$

where $\Delta x$ and $\Delta y$ denote the discrete integration intervals, and $N_{1}$ and $N_{2}$ are the number of intervals along $x$ and $y$ coordinates. The reference solution can be the exact steady-state PDF or the steady-state PDF from Monte Carlo simulations.

\subsection{Progressively Iterative Procedure}

The method of weighted residue has an interesting property. When the assumed PDF solution $\bar{p}(x, y)$ and the weighting function $p_{m}(x, y)$ are far away from the true solution, the iterative procedure may not converge to a sufficiently accurate solution of the steady-state PDF. This is often the case when the system is strongly nonlinear.

One way to overcome this difficulty for strongly nonlinear systems is to choose $p_{m}(x, y)$ as the PDF solution of a weakly nonlinear system, and progressive increasing the nonlinear parameter when we apply the iterative method of weighted residue to find the PDF solutions.

\section{$3 \quad$ Examples}

\section{Example 1}

As the first example, we consider the Duffing oscillator with multiple stable equilibrium states under external Gaussian white noise excitation. The equations of motion in the state space are

$$
\begin{aligned}
& \frac{d X}{d t}=Y \\
& \frac{d Y}{d t}=-\beta Y-\alpha_{1} X+\alpha_{3} X^{3}-\alpha_{5} X^{5}+W_{1}(t)
\end{aligned}
$$

where $\beta, \alpha_{1}, \alpha_{3}$ and $\alpha_{5}$ are positive constants. $W(t)$ denotes the Gaussian white noise excitation with intensity $2 D_{1}$. The drift and diffusion coefficients of the reduced FPK equation 
are given by

$$
\begin{aligned}
& m_{1}=y \\
& m_{2}=\beta y+\alpha_{1} x-\alpha_{3} x^{3}+\alpha_{5} x^{5}, \\
& b_{22}=D_{1} .
\end{aligned}
$$

We select the parameters $\beta=0.1, \alpha_{1}=1.0, \alpha_{3}=3.5, \alpha_{5}=1.0, D_{1}=0.1, \varepsilon_{0}=10^{-3}$, and $n=6$. Note that this system is strongly nonlinear because of the relatively large value of $\alpha_{3}$. We apply the progressive iterative procedure to calculate the PDF. We start with $\alpha_{3}=2.2$ and apply the iterative method of weighted residue to compute the steady-state PDF $\bar{p}(x, y)$ by selecting a Gaussian PDF for $p_{m}(x, y)$. Then, we let $p_{m}(x, y)$ be the obtained approximate steady-state PDF and apply the iterative method again to compute $\bar{p}(x, y)$ for $\alpha_{3}=2.3$. Progressively, the steady-state PDF $\bar{p}(x, y)$ for $\alpha_{3}=3.5$ is obtained as,

$$
\begin{aligned}
\bar{p}(x, y) & =C_{0} \exp \left[-0.5 x^{2}+2.483643640 \times 10^{-9} x y\right. \\
& -0.4999999761 y^{2}+0.8750000459 x^{4}-0.1666666728 x^{6} \\
& -7.642985230 \times 10^{-10} x^{3} y-1.240048578 \times 10^{-8} x^{2} y^{2} \\
& -8.332558060 \times 10^{-10} x y^{3}-1.583955822 \times 10^{-9} y^{4} \\
& +6.244126235 \times 10^{-11} x^{5} y+1.184985111 \times 10^{-9} x^{4} y^{2} \\
& +1.290148585 \times 10^{-10} x^{3} y^{3}+5.215769425 \times 10^{-10} x^{2} y^{4} \\
& \left.+3.576490422 \times 10^{-11} x y^{5}-2.717927530 \times 10^{-11} y^{6}\right] .
\end{aligned}
$$

After neglecting the terms with coefficients of the order $10^{-8}$ and higher, the solution in Equation (16) is in agreement with the exact steady-state PDF,

$$
p_{\text {ext }}(x, y)=C \exp \left[-\left(\frac{\beta y^{2}}{2}+\frac{\alpha_{1} x^{2}}{2}-\frac{\alpha_{3} x^{4}}{4}+\frac{\alpha_{5} x^{6}}{6}\right)\right] .
$$

Table 1 shows the convergence of the iterative method for every $\alpha_{3}$. It is seen that the convergence is very fast for all $\alpha_{3}$ values.

\section{Example 2}

Consider an energy dependent system with parametric excitation governed by the following stochastic differential equations [7],

$$
\begin{aligned}
& \frac{d X}{d t}=Y, \\
& \frac{d Y}{d t}=[-\beta \xi(\lambda) Y-g(X)]+\eta(\lambda) W_{1}(t),
\end{aligned}
$$

where $\beta>0$ is a constant, $W_{1}(t)$ denotes the Gaussian white noise excitation with intensity $2 D_{1}, g(X)$ is the linear or nonlinear spring force, $\xi(\lambda)$ and $\eta(\lambda)$ are arbitrary functions, and 
$\lambda$ is the total mechanical energy,

$$
\lambda=\frac{1}{2} Y^{2}+\int_{0}^{X} g(x) d x
$$

The drift and diffusion terms are given by,

$$
\begin{aligned}
& m_{1}=y \\
& m_{2}=-\beta \xi(\lambda) y-g(x)+D_{1} \eta \frac{\partial \eta}{\partial y} \\
& b_{22}=D_{1}[\eta(\lambda)]^{2}
\end{aligned}
$$

The exact steady-state PDF of the response is known,

$$
p_{\text {ext }}(x, y)=\frac{C}{\left[b_{22}\right]^{1 / 2}} \exp \left[-\frac{1}{D_{1}} \int_{0}^{\lambda} \frac{\beta \xi(\lambda)}{[\eta(\lambda)]^{2}} d x\right]
$$

In order to illustrate the proposed method, we consider a specific case with $\beta=0.3, \varepsilon_{0}=$ $10^{-3}, \xi(\lambda)=\lambda, g(x)=x, \eta(\lambda)=\frac{1}{\sqrt{\lambda}}$ and $D_{1}=0.1$. Then, the exact solution reads,

$$
p_{\text {ext }}(x, y)=C\left(x^{2}+y^{2}\right)^{1 / 2} \exp \left[-\frac{\beta}{24 D_{1}}\left(x^{2}+y^{2}\right)^{3}\right] \text {. }
$$

Let $p_{m}(x, y)$ be a Gaussian PDF given by,

$$
p_{m}(x, y)=C_{0} \exp \left[-\frac{\beta}{2 D_{1}}\left(x^{2}+y^{2}\right)\right]
$$

The iterative method with $n=8$ starts to compute the steady-state PDF for $\beta=0.1$ and progresses to $\beta=0.3$. The solution is given by 


$$
\begin{aligned}
\bar{p}(x, y) & =0.1904809311\left(x^{2}+y^{2}\right)^{.5000000002} \exp \left[-4.435044876 \times 10^{-8} x y^{5}\right. \\
& +3.812579917 \times 10^{-9} x^{7} y+1.379244061 \times 10^{-8} x^{3} y^{5} \\
& +7.368077272 \times 10^{-8} x^{3} y-9.055975438 \times 10^{-9} x^{6} y^{2} \\
& +6.261100113 \times 10^{-9} x y^{7}-5.862813841 \times 10^{-8} x y \\
& +1.196291826 \times 10^{-8} x^{5} y^{3}-1.644482745 \times 10^{-9} x^{4} y^{4} \\
& -2.136351359 \times 10^{-8} x^{2} y^{2}-2.968863339 \times 10^{-8} x^{5} y \\
& +9.483932968 \times 10^{-8} x y^{3}+6.782634374 \times 10^{-9} x^{2} y^{6} \\
& -6.771755571 \times 10^{-8} x^{3} y^{3}-6.912070755 \times 10^{-8} x^{4} \\
& +4.111977875 \times 10^{-8} y^{4}+3.011814811 \times 10^{-9} x^{8} \\
& -4.316925205 \times 10^{-9} x^{8}-1.610709756 \times 10^{-8} y^{2} \\
& -0.3750000194 x^{2} y^{4}+5.349592654 \times 10^{-8} x^{2} \\
& \left.-0.3749999639 x^{4} y^{2}-0.1250000210 y^{6}-0.1249999693 x^{6}\right]
\end{aligned}
$$

After neglecting the terms with coefficients of the order $10^{-8}$ and higher, the solution in Equation (24) matches with the exact steady-state PDF perfectly. In Table 2, the convergence of the iterative method for different values of $\beta$ is presented. The fast convergence is also found for every $\beta$.

\section{Example 3}

Finally, we consider Dimentberg's system [5]. The equation of motion in the state space is given by

$$
\begin{aligned}
& \frac{d X}{d t}=Y, \\
& \frac{d Y}{d t}=-2 \alpha Y\left[1+W_{1}(t)\right]-\beta_{1} Y\left(X^{2}+\frac{Y^{2}}{\Omega^{2}}\right)-\Omega^{2} X\left[1+W_{2}(t)\right]+W_{3}(t),
\end{aligned}
$$

where $\beta_{1} \geq 0, \alpha>0$, and $W_{i}(t)(i=1,2,3)$ denote the independent Gaussian white noise excitations with intensities $2 D_{i}$. The drift and diffusion coefficients of the FPK equation are given by,

$$
\begin{aligned}
& m_{1}=y \\
& m_{2}=2 \alpha y+\beta_{1} y\left(x^{2}+\frac{y^{2}}{\Omega^{2}}\right)+\Omega^{2} x-4 \alpha^{2} D_{1} y, \\
& b_{22}=4 D_{1} \alpha^{2} y^{2}+D_{2} \Omega^{4} x^{2}+D_{3} .
\end{aligned}
$$

Case $14 D_{1} \alpha^{2}=D_{2} \Omega^{4}$

In this case, the reduced FPK equation has an exact solution. Let $\alpha=0.25, \beta_{1}=0.1$, $D_{1}=D_{3}=0.1, D_{2}=0.025$, and, $\Omega=1$. We have 


$$
\begin{aligned}
p_{\text {ext }}(x, y) & =C \exp \left[-\beta\left(x^{2}+\frac{y^{2}}{\Omega^{2}}\right)-(\eta-\varkappa \beta) \ln \left(x^{2}+\frac{y^{2}}{\Omega^{2}}+\varkappa\right)\right] \\
& =C \exp \left[-4\left(x^{2}+y^{2}\right)-4.5 \ln \left(x^{2}+y^{2}+4\right)\right]
\end{aligned}
$$

where $\varkappa=\frac{D_{3}}{D_{2} \Omega^{4}}=4, \eta=\frac{2 \alpha}{D_{2} \Omega^{2}}+\frac{1}{2}=20.5, \beta=\frac{\beta_{1}}{D_{2} \Omega^{2}}=4$ and $C$ is the normalization constant. Clearly, the steady-state PDF of system (25) is not in the form of exponential polynomials.

Choose a Gaussian PDF for $p_{m}(x, y)$ as

$$
p_{m}(x, y)=C \exp \left[-\frac{\beta}{D_{3}}\left(x^{2}+\frac{y^{2}}{\Omega^{2}}\right)\right] .
$$

We have taken $\varepsilon_{0}=10^{-3}$ and $n=6$. The iterative method of weighted residue gives the following solution after 2 iterations,

$$
\begin{aligned}
\bar{p}(x, y) & =0.00005125363170 \exp \left[-4.000026413 x^{2}-1.690501939 \times 10^{-8} x y\right. \\
& -4.000026444 y^{2}+0.000003059301744 x^{4} \\
& +1.092201787 \times 10^{-7} x^{3} y+0.000006183873995 x^{2} y^{2} \\
& +2.606680097 \times 10^{-8} x y^{3}+0.000003168021220 y^{4} \\
& -2.859245324 \times 10^{-7} x^{6}-1.161179229 \times 10^{-7} x^{5} y \\
& -8.626914653 \times 10^{-7} x^{4} y^{2}-1.216582078 \times 10^{-7} x^{3} y^{3} \\
& -9.682102840 \times 10^{-7} x^{2} y^{4}-1.214194033 \times 10^{-8} x^{1} y^{5} \\
& \left.-3.632357122 \times 10^{-7} y^{6}-4.499894038 \ln \left(0.0025 y^{2}+0.0025 x^{2}+0.1\right)\right] .
\end{aligned}
$$

After neglecting the terms with coefficients of the order $10^{-7}$ and higher, we see that this solution matches extremely well with the exact steady-state PDF in Equation (27).

An interesting observation to make is that all the terms in the polynomial of order higher than 2 for this and of order higher than 6 for Example 2 are practically zero, in agreement with the exact solutions. It appears that when the assumed PDF solution matches the form of the true solution including the logarithmic term $\ln \left(b_{22}\right)$, the order of the polynomial can be finite in order to converge to the exact solution.

Next, we demonstrate when there is a mismatch in the function form of the PDF, the order of the polynomials needs to be high enough. Let $\bar{p}(x, y)$ be in the form without the logarithmic term,

$$
\bar{p}(x, y)=C_{0} \exp \left[\varphi\left(x, y, c_{i j}\right)\right]
$$

where $\varphi\left(x, y, c_{i j}\right)$ is defined in Equation (5). The steady-state PDF with the order of polynomials $n=6,8,10$ and 12 are computed with the proposed iterative method. For the sake of space, we only show the solution for $n=6$ below, 


$$
\begin{aligned}
\bar{p}(x, y) & =1.56857817 \exp \left[-4.870681657 x^{2}-0.1827437611 x y\right. \\
& -4.880327404 y^{2}-0.2612748227 x^{4}+0.5337262697 x^{3} y \\
& -0.7642525316 x^{2} y^{2}+0.6819686086 x y^{3}-0.3394666199 y^{4} \\
& +0.3071249386 x^{6}-0.4107765872 x^{5} y+1.176650210 x^{4} y^{2} \\
& -1.038374624 x^{3} y^{3}+1.471012318 x^{2} y^{4} \\
& \left.-0.6527673193 x y^{5}+0.4687087249 y^{6}\right]
\end{aligned}
$$

Note that the coefficients of all the terms in the polynomial are of the same order of magnitude. Table 3 shows the convergence rate and the errors of the steady-state PDFs $\bar{p}(x, y)$ with $n=6,8,10$ and 12 compared to the exact solution in Equation (27). The data in Table 3 show that the order $n$ of the polynomial must be large enough in order for $\bar{p}(x, y)$ to converge to the true solution. However, the large value of $n$ will require far more intensive computations.

Case $24 D_{1} \alpha^{2} \neq D_{2} \Omega^{4}$.

The reduced FPK equation has no exact solution in this case. Suppose that $\alpha=0.8, \beta_{1}=1$, $D_{i}=1, \Omega=1$ and $\varepsilon_{0}=10^{-3}$. We let $p_{m}(x, y)$ be the PDF obtained with the method of stochastic averaging given by,

$$
\begin{aligned}
p_{\text {ave }}(x, y) & =8.351251535 \exp \left[-0.2304147466 x^{2}-0.2304147466 y^{2}\right. \\
& \left.-0.8523434348 \ln \left(108.5 x^{2}+108.5 y^{2}+50\right)\right] .
\end{aligned}
$$

We obtain the steady-state PDF as assumed in Equation (4) with $n=4$ after 6 iterations,

$$
\begin{aligned}
\bar{p}(x, y) & =0.2645931404 \exp \left[-0.2427942155 x^{2}+0.016612169 x y\right. \\
& -0.1586363505 y^{2}-0.0198785238 x^{4}-0.00016793712 x^{3} y \\
& -0.0329402734 x^{2} y^{2}+0.0067945304 x y^{3}-0.0008684332 y^{4} \\
& \left.-0.8441757887 \ln \left(2.56 y^{2}+x^{2}+1\right)\right]
\end{aligned}
$$

For comparison, the steady-state PDF as assumed in Equation (30) is also obtained with $n=6$ after 16 iterations,

$$
\begin{aligned}
\bar{p}(x, y) & =0.5351325151 \exp \left[-0.8317891371 x^{6}+0.5768585612 x^{5} y\right. \\
& -0.1618104089 x^{4} y^{2}+0.0436025228 x^{3} y^{3}-0.02017895631 x^{2} y^{4} \\
& +0.0035169154 x y^{5}-0.0107331756 y^{6}+2.824045797 x^{4} \\
& -1.522128260 x^{3} y+0.4120185815 x^{2} y^{2}-0.0922210711 x y^{3} \\
& +0.1683768861 y^{4}-4.157036701 x^{2} \\
& \left.+1.209142390 x y-1.329380163 y^{2}\right] .
\end{aligned}
$$

To check the accuracy of the analytical approximations, we have conducted extensive Monte Carlo simulations to compute the steady-state PDF of the system. Figure 1(a) shows the 
steady-state PDF obtained from Monte Carlo simulations with $10^{6}$ points. Figure $1(\mathrm{~b})$ denotes the closed-form solution in Equation (33). The error of the PDF solution as defined in Equation (13) is 0.01802. Figure 1(c) plots the solution in Equation (32) obtained with the method of stochastic averaging. Its error is 0.0443. Figure $1(\mathrm{~d})$ draws the exponential polynomial solution in Equation (34). Its error is 0.2410 .

In Figure 2, the marginal distribution densities of $p_{1}(x)$ and $p_{1}(y)$ from the Monte Carlo simulations and closed-form solutions in Equations (33-34) are illustrated. The root mean squared (RMS) error between the analytical results and Monte Carlo data are also shown in the figures. Clearly, the solution in Equation (33) obtained with the proposed method is in excellent agreement with the Monte Carlo simulation, and has the best accuracy among the three closed-form solutions.

\section{Conclusions}

We have developed a new method to find the closed-form solution of the reduced FPK equation of single-degree-of-freedom nonlinear systems under external and parametric Gaussian white noise excitations. The assumed PDF solution is in the form of exponential polynomials with a logarithmic term to account for parametric excitations. The method of weighted residue is employed to determine the unknown parameters in the assumed solution. An iterative procedure is developed to improve the accuracy of the solution obtained with the method of weighted residue. A progressively iterative procedure for finding the PDF solutions of strongly nonlinear systems is also developed. The proposed method can lead to the exact solution when it exists, and can yield a highly accurate solution as compared with Monte Carlo simulations when the exact solution is not available. Numerical results of three difficult examples have been presented to demonstrate the method.

\section{Acknowledgements}

This work is supported by the Natural Science Foundation of China through the grants (11172197, 11332008 and 11572215), by the Natural Science Foundation of Tianjin through a key-project grant, and Fujian Province through the grant (2014J01014), and by Research Award Fund for Outstanding Young Researcher in Higher Education Institutions of Fujian Province. The first author would like to thank the China Scholarship Council for sponsoring his studies at University of California, Merced through the grant (201408350008). 


\section{References}

[1] G. Q. Cai, Y. K. Lin, On exact stationary solutions of equivalent non-linear stochastic systems, International Journal of Non-Linear Mechanics 23 (4) (1988) 315-325.

[2] T. K. Caughey, Nonlinear theory of random vibrations, in: C.-S. Yih (Ed.), Advances in Applied Mechanics, Vol. 11, Elsevier, 1971, pp. 209-253.

[3] C. W. Gardiner, Handbook of Stochastic Methods for Physics, Chemistry and Natural Sciences, Springer, Berlin, 1983.

[4] T. K. Caughey, F. Ma, The exact steady-state solution of a class of non-linear stochastic systems, International Journal of Non-Linear Mechanics 17 (3) (1982) 137-142.

[5] M. F. Dimentberg, An exact solution to a certain non-linear random vibration problem, International Journal of Non-Linear Mechanics 17 (4) (1982) 231-236.

[6] Y. Yong, Y. K. Lin, Exact stationary-response solution for second order nonlinear systems under parametric and external white-noise excitations, Journal of Applied Mechanics 54 (2) (1987) 414-418.

[7] W. Q. Zhu, Exact solutions for stationary responses of several classes of nonlinear systems to parametric and/or external white noise excitations, Applied Mathematics and Mechanics 11 (2) (1990) 165-175.

[8] W. Q. Zhu, G. Q. Cai, Y. K. Lin, On exact stationary solutions of stochastically perturbed Hamiltonian systems, Probabilistic Engineering Mechanics 5 (2) (1990) 84-87.

[9] Y. K. Lin, G. Q. Cai, Probabilistic Structural Dynamics, Advanced Theory and Applications, McGraw-Hill, New York, 1995.

[10] R. S. Langley, A finite element method for the statistics of non-linear random vibration, Journal of Sound and Vibration 101 (1) (1985) 41-54.

[11] B. F. Spencer, L. A. Bergman, On the numerical solution of the Fokker-Planck equation for nonlinear stochastic systems, Nonlinear Dynamics 4 (4) (1993) 357-372.

[12] L.-C. Shiau, T.-Y. Wu, A finite-element method for analysis of a non-linear system under stochastic parametric and external excitation, International Journal of Non-Linear Mechanics 31 (2) (1996) 193-201.

[13] A. Masud, L. A. Bergman, Application of multi-scale finite element methods to the solution of the Fokker-Planck equation, Computer Methods in Applied Mechanics and Engineering 194 (12-16) (2005) 1513-1526.

[14] P. Kumar, S. Narayanan, S. Gupta, Finite element solution of Fokker-Planck equation of nonlinear oscillators subjected to colored non-Gaussian noise, Probabilistic Engineering Mechanics 38 (2014) 143-155.

[15] M. P. Zorzano, H. Mais, L. Vazquez, Numerical solution of two dimensional Fokker-Planck equations, Applied Mathematics and Computation 98 (2-3) (1999) 109-117. 
[16] H. Fukushima, Y. Uesaka, Y. Nakatani, N. Hayashi, Numerical solutions of the Fokker-Planck equation by the finite difference method for the thermally assisted reversal of the magnetization in a single-domain particle, Journal of Magnetism and Magnetic Materials 242-245 (Part 2) (2002) 1002-1004.

[17] P. Kumar, S. Narayanan, Solution of Fokker-Planck equation by finite element and finite difference methods for nonlinear systems, Sadhana 31 (4) (2006) 445-461.

[18] M. F. Wehner, W. G. Wolfer, Numerical evaluation of path-integral solutions to Fokker-Planck equations, Physical Review A 27 (5) (1983) 2663-2670.

[19] A. Naess, V. Moe, Efficient path integration methods for nonlinear dynamic systems, Probabilistic Engineering Mechanics 15 (2) (2000) 221-231.

[20] P. Kumar, S. Narayanan, Modified path integral solution of Fokker-Planck equation: Response and bifurcation of nonlinear systems, Journal of Computational and Nonlinear Dynamics 5 (1) (2009) 011004-12.

[21] W. V. Wedig, Iterative schemes for stability problems with non-singular Fokker-Planck equations, International Journal of Non-Linear Mechanics 31 (5) (1996) 707-715.

[22] M. Kumar, S. Chakravorty, J. L. Junkins, A homotopic approach to domain determination and solution refinement for the stationary Fokker-Planck equation, Probabilistic Engineering Mechanics 24 (3) (2009) 265-277.

[23] K. Sobczyk, J. Trbicki, Maximum entropy principle and nonlinear stochastic oscillators, Physica A: Statistical Mechanics and its Applications 193 (3-4) (1993) 448-468.

[24] R. J. Chang, Maximum entropy approach for stationary response of nonlinear stochastic oscillators, Journal of Applied Mechanics 58 (1) (1991) 266-271.

[25] S. A. El-Wakil, A. Elhanbaly, M. A. Abdou, Solution of Fokker-Planck equation by means of maximum entropy approach, Journal of Quantitative Spectroscopy and Radiative Transfer 69 (1) (2001) 41-48.

[26] J. D. Atkinson, Eigenfunction expansions for randomly excited non-linear systems, Journal of Sound and Vibration 30 (2) (1973) 153-172.

[27] J. P. Johnson, R. A. Scott, Extension of eigenfunction-expansion solutions of a Fokker-Planck equation - I. First order system, International Journal of Non-Linear Mechanics 14 (5-6) (1979) $315-324$.

[28] J. P. Johnson, R. A. Scott, Extension of eigenfunction-expansion solutions of a Fokker-Planck equation - II. Second order system, International Journal of Non-Linear Mechanics 15 (1) (1980) $41-56$.

[29] T. Blum, A. J. McKane, Variational schemes in the Fokker-Planck equation, Journal of Physics A: Mathematical and General 29 (9) (1996) 1859-1872.

[30] Q. Liu, H. G. Davies, The non-stationary response probability density functions of non-linearly damped oscillators subjected to white noise excitations, Journal of Sound and Vibration 139 (3) (1990) 425-435. 
[31] G. Muscolino, G. Ricciardi, M. Vasta, Stationary and non-stationary probability density function for non-linear oscillators, International Journal of Non-Linear Mechanics 32 (6) (1997) 1051-1064.

[32] U. von Wagner, W. V. Wedig, On the calculation of stationary solutions of multi-dimensional Fokker-Planck equations by orthogonal functions, Nonlinear Dynamics 21 (3) (2000) 289-306.

[33] G.-K. Er, An improved closure method for analysis of nonlinear stochastic systems, Nonlinear Dynamics 17 (3) (1998) 285-297.

[34] G.-K. Er, A consistent method for the solution to reduced FPK equation in statistical mechanics, Physica A: Statistical Mechanics and its Applications 262 (1-2) (1999) 118-128.

[35] G.-K. Er, Exponential closure method for some randomly excited non-linear systems, International Journal of Non-Linear Mechanics 35 (1) (2000) 69-78.

[36] W. Martens, U. von Wagner, V. Mehrmann, Calculation of high-dimensional probability density functions of stochastically excited nonlinear mechanical systems, Nonlinear Dynamics 67 (3) (2012) 2089-2099.

[37] C. S. Manohar, Methods of nonlinear random vibration analysis, Sadhana 20 (2-4) (1995) $345-371$.

[38] H. Risken, T. Frank, The Fokker-Planck equation methods of solution and application, Springer, 1996.

[39] H. T. Zhu, G. K. Er, V. P. Iu, K. P. Kou, EPC procedure for PDF solution of non-linear oscillators excited by poisson white noise, International Journal of Non-Linear Mechanics 44 (3) (2009) 304-310.

[40] H. T. Zhu, G. K. Er, V. P. Iu, K. P. Kou, Probabilistic solution of nonlinear oscillators excited by combined Gaussian and Poisson white noises, Journal of Sound and Vibration 330 (12) (2011) 2900-2909.

[41] H. T. Zhu, Multiple-peak probability density function of non-linear oscillators under Gaussian white noise, Probabilistic Engineering Mechanics 31 (2013) 46-51.

[42] H. T. Zhu, Response of a vibro-impact Duffing system with a randomly varying damping term, International Journal of Non-Linear Mechanics 65 (2014) 53-62.

[43] H. T. Zhu, Stochastic response of vibro-impact Duffing oscillators under external and parametric Gaussian white noises, Journal of Sound and Vibration 333 (3) (2014) 954-961.

[44] G.-K. Er, Probabilistic solutions of some multi-degree-of-freedom nonlinear stochastic dynamical systems excited by filtered Gaussian white noise, Computer Physics Communications 185 (4) (2014) 1217-1222.

[45] M. D. Paola, A. Sofi, Approximate solution of the Fokker-Planck-Kolmogorov equation, Probabilistic Engineering Mechanics 17 (4) (2002) 369-384. 
Table 1

Number of iterations of the method of weighted residue to converge for each $\alpha_{3}$ of Example 1.

\begin{tabular}{|c|c|c|c|c|c|c|}
\hline$\alpha_{3}$ & 2.2 & 2.3 & 2.5 & 2.8 & 3.0 & 3.5 \\
\hline \hline Iterations & 2 & 2 & 1 & 1 & 1 & 1 \\
\hline
\end{tabular}

Table 2

Number of iterations of the method of weighted residue to converge for each $\beta$ of Example 2 .

\begin{tabular}{|c|c|c|c|}
\hline$\beta$ & 0.1 & 0.2 & 0.3 \\
\hline \hline Iterations & 2 & 1 & 1 \\
\hline
\end{tabular}

Table 3

Number of iterations of the method of weighted residue to converge and the error of the steady-state PDF for different orders of polynomial in the solution in Equation (30) of Example 3.

\begin{tabular}{|c|c|c|c|c|}
\hline$n$ & 6 & 8 & 10 & 12 \\
\hline \hline$\Delta p$ & 0.0185 & 0.0539 & $2.88 \times 10^{-6}$ & $8.79 \times 10^{-7}$ \\
\hline Iterations & 12 & 16 & 4 & 2 \\
\hline
\end{tabular}



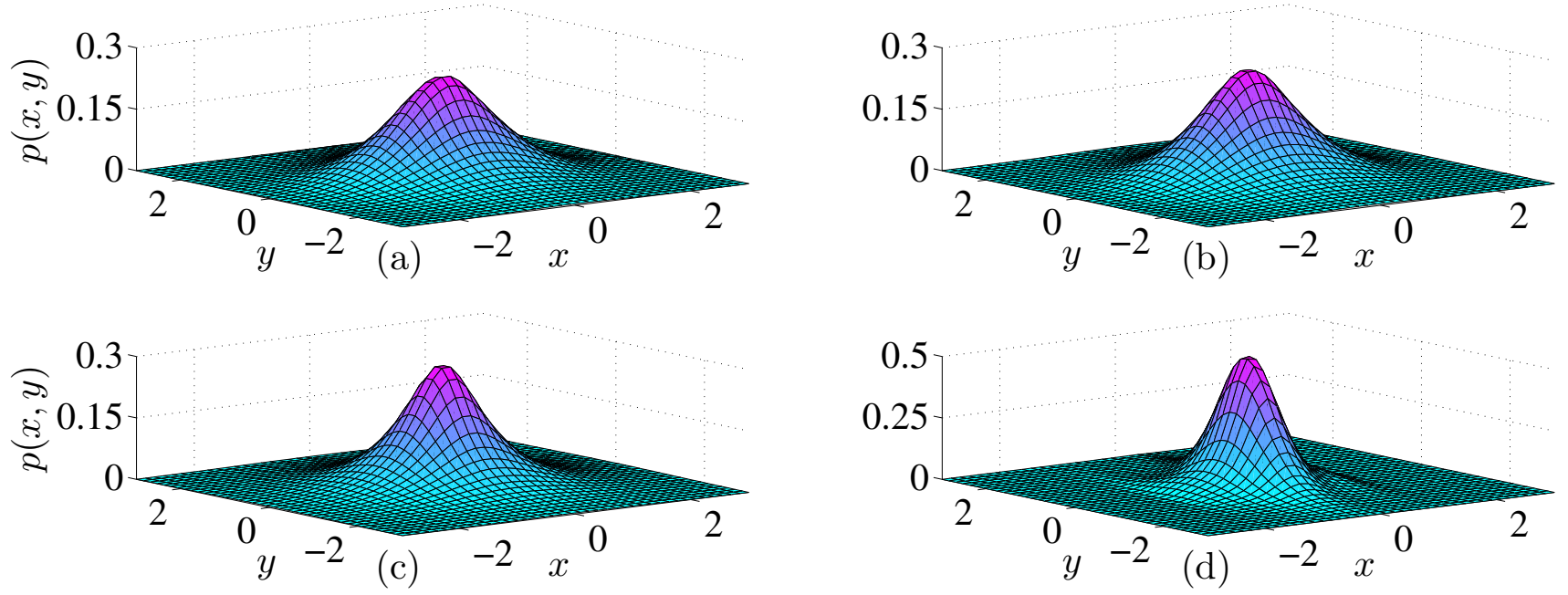

Fig. 1. The steady-state PDF of Dimentberg's system with parameters $\alpha=0.8, \beta=1$ and $D_{i}=$ 1.0.(a) The Monte Carlo simulation. (b) The solution in Equation (33). (c) The solution with the method of stochastic averaging in Equation (32). (d) The solution with the method of exponential polynomial in Equation (34).
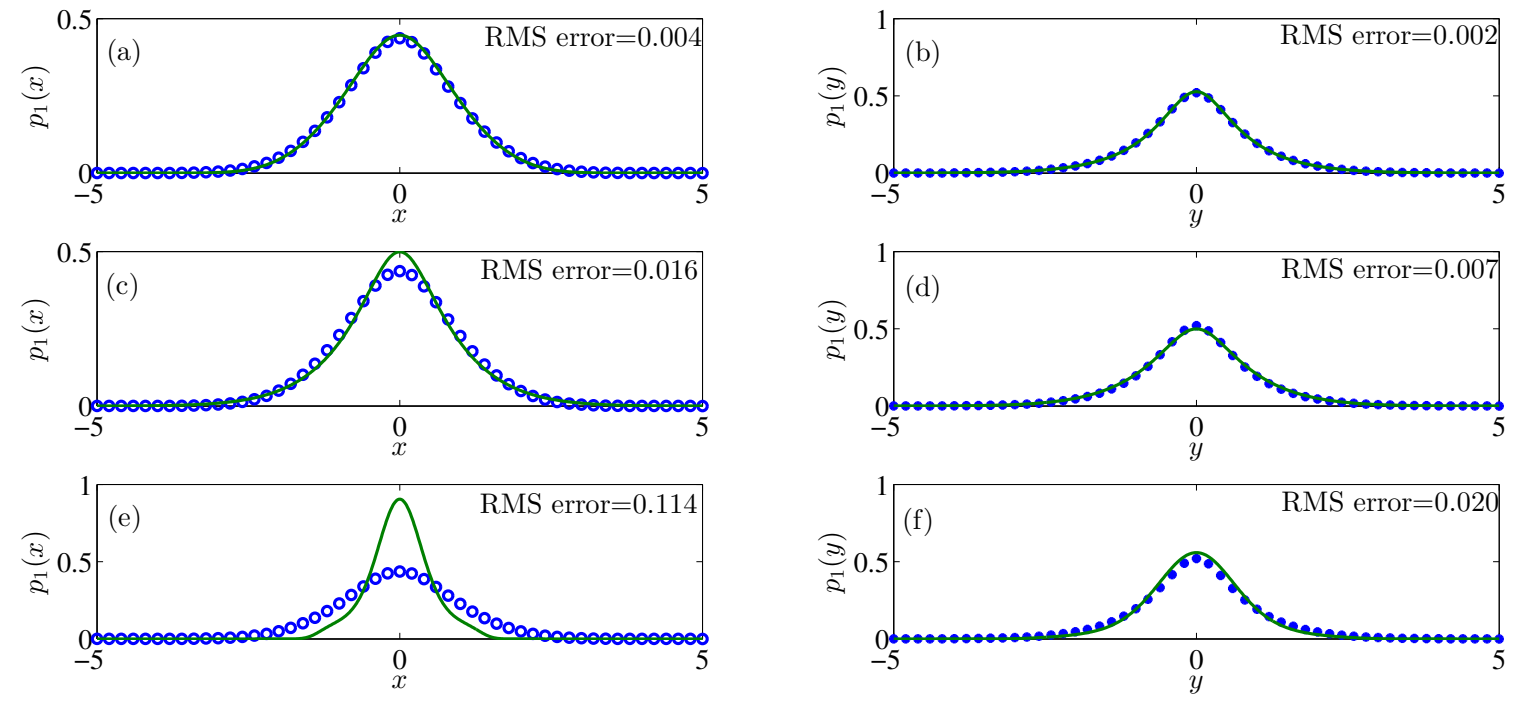

Fig. 2. The marginal probability densities of $p_{1}(x)$ and $p_{1}(y)$ of Dimentberg's system obtained from the corresponding joint PDFs. Symbols $\left(\mathrm{o},{ }^{*}\right)$ denote the Monte Carlo data. Solid line denotes the analytical solutions. (a) and (b): From Equation (33). (c) and (d): From Equation (32). (e) and (f): From Equation (34). 